\title{
Lack of Difference Among Terlipressin, Somatostatin, and Octreotide in the Control of Acute Gastroesophageal Variceal Hemorrhage
}

\author{
Yeon Seok Seo, ${ }^{1}$ Soo Young Park, ${ }^{2}$ Moon Young Kim, ${ }^{3}$ Ju Hyun Kim, ${ }^{4}$ Jun Yong Park, ${ }^{5}$ Hyung Joon Yim, ${ }^{1}$ \\ Byoung Kuk Jang, ${ }^{6}$ Hong Soo Kim, ${ }^{7}$ Taeho Hahn, ${ }^{8}$ Byung Ik Kim, ${ }^{9}$ Jeong Heo, ${ }^{10}$ Hyonggin An, ${ }^{11}$ \\ Won Young Tak, ${ }^{2}$ Soon Koo Baik, ${ }^{3,12}$ Kwang Hyub Han, ${ }^{5,12}$ Jae Seok Hwang, ${ }^{6}$ Sang Hoon Park, ${ }^{8}$ \\ Mong Cho, ${ }^{10}$ and Soon Ho $\mathrm{Um}^{1,12}$
}

\begin{abstract}
Vasoactive drugs are recommended to be started as soon as possible in suspected variceal bleeding, even before diagnostic endoscopy. However, it is still unclear whether the therapeutic efficacies of the various vasoactive drugs used are comparable. The aim of this prospective, multicenter, randomized, noninferiority trial was to characterize the effects of terlipressin, somatostatin, and octreotide when they are initiated before endoscopic treatment in patients with acute variceal bleeding. Patients with liver cirrhosis and significant upper gastrointestinal bleeding were randomly assigned to receive early administration of terlipressin, somatostatin, or octreotide, followed by endoscopic treatment. Patients with nonvariceal bleeding were excluded after endoscopy. The primary endpoint was 5-day treatment success, defined as control of bleeding without rescue treatment, rebleeding, or mortality, with a noninferiority margin of 0.1 . In total, 780 patients with variceal bleeding were enrolled: 261 in the terlipressin group; 259 in the somatostatin group; and 260 in the octreotide group. At the time of initial endoscopy, active bleeding was noted in $43.7 \%, 44.4 \%$, and $43.5 \%$ of these patients, respectively $(P=0.748)$, and treatment success was achieved by day 5 in $86.2 \%, 83.4 \%$, and $83.8 \%(P=0.636)$, with similar rates of control of bleeding without rescue treatment $(89.7 \%, 87.6 \%$, and $88.1 \% ; P=0.752)$, rebleeding $(3.4 \%, 4.8 \%$, and $4.4 \% ; P=0.739)$, or mortality $(8.0 \%, 8.9 \%$, and $8.8 \%$; $P=0.929)$. The absolute values of the lower bound of confidence intervals for terlipressin versus somatostatin, terlilpressin versus octreotide, and octreotide versus somatostatin were 0.095 , 0.090, and 0.065, respectively. Conclusion: Hemostatic effects and safety did not differ significantly between terlipressin, somatostatin, and octreotide as adjuvants to endoscopic treatment in patients with acute gastroesophageal variceal bleeding. (HEPATOLOGY 2014;60:954-963)
\end{abstract}

See Editorial on Page 789

A cute variceal bleeding is one of the critical complications in patients with liver cirrhosis. ${ }^{1}$ Although the mortality rate has decreased significantly during the past several decades as a result of remarkable improvements in diagnostic and therapeutic modalities for its management, acute variceal bleeding remains a leading cause of death in patients with cirrhosis. ${ }^{1,2}$ Therefore, appropriate, effective treatments should be performed promptly in these patients.

Abbreviations: AEs, adverse events; AST, aspartate aminotransferase; BP, blood pressure; CI, confidence interval; EIS, endoscopic injection sclerotherapy; EVs, esophageal varices; EVL, endoscopic variceal ligation; EVO, endoscopic variceal obturation; PH, portal hypertension; GOVs, gastroesophageal varices; GVs, gastric varices; Hb, hemoglobin; HCC, hepatocellular carcinoma; Hct, hematocrit; IGVs, isolated gastric varices; INR, international normalized ratio; IV, intravenous; MELD, Model for End-stage Liver Disease; OR, odds ratio; TO, time of admission at the first hospital; TIPS, transjugular intrahepatic portosystemic shunt.

From the ${ }^{1}$ Department of Internal Medicine, Korea University College of Medicine, Seoul, Korea; ${ }^{2}$ Kyungpook National University School of Medicine, Daegu, Korea; ${ }^{3}$ Yonsei University Wonju College of Medicine, Wonju, Korea; ${ }^{4}$ Gachon Medical School, Gil Medical Center, Incheon, Korea; ${ }^{5}$ Yonsei University College of Medicine, Seoul, Korea; ${ }^{6}$ Keimyung University College of Medicine, Daegu, Korea; ${ }^{7}$ Soonchunhyang University College of Medicine, Cheonan, Korea; ${ }^{8}$ Hallym University College of Medicine, Anyang, Korea; ${ }^{9}$ Sungkyunkwan University College of Medicine, Kangbuk Samsung Hospital, Seoul, Korea; ${ }^{10}$ Pusan National University College of Medicine, Busan, Korea; ${ }^{11}$ Department of Biostatistics, Korea University College of Medicine, Seoul, Korea; ${ }^{12}$ Liver Cirrhosis Clinical Research Center, Seoul, Korea.

Received May 16, 2013; accepted January 5, 2014.

This study was supported by a grant from the Ministry of Health, Welfare and Family Affairs, Republic of Korea (no. A050021). 
Three vasoactive drugs-terlipressin, somatostatin, and octreotide-play a role in the control of variceal bleeding by reducing portal blood flow and portal pressure. ${ }^{3,4}$ Previous studies showed that use of vasoactive drugs was associated with a significantly lower risk of mortality and transfusion requirements as well as an improved control of bleeding. ${ }^{5,6}$ In particular, treatment response in patients with variceal bleeding was significantly better when combining vasoactive drugs with endoscopic therapy than with pharmacological or endoscopic monotherapy. ${ }^{7-11}$ Therefore, current practice guidelines recommend the combination of pharmacological therapy and endoscopic therapy as the standard treatment for acute variceal bleeding, particularly the early administration of vasoactive drugs even before endoscopy in suspected variceal bleeding. ${ }^{12-14}$ Regarding the methods of endoscopic therapy, several studies suggested that the probability of rebleeding from esophageal varices (EVs) is significantly lower for endoscopic variceal ligation (EVL) than for endoscopic injection sclerotherapy (EIS). ${ }^{15,16}$

There is no preference when selecting one of these three vasoactive drugs because their efficacies seem to be comparable, although some researchers recommend terlipressin as the first choice ${ }^{17}$ because it is the only drug that was found to improve the survival in placebo-controlled trials ${ }^{18,19}$ and a meta-analysis. ${ }^{5}$ However, it is unclear whether the earlier results could be directly applied to the current clinical situations, because most patients in the previous studies were treated with vasoactive drugs alone or with vasoactive drugs plus EIS whereas EVL is now primarily recommended for the endoscopic control of esophageal variceal bleeding in combination with vasoactive drugs. In addition, there are some concerns regarding the hemostatic efficacy of octreotide because it was ineffective in the setting of monotherapy in a randomized, controlled trial, ${ }^{20}$ and desensitization of octreotide was reported in patients with cirrhosis with portal hypertension $(\mathrm{PH}){ }^{21}$

The present multicenter, prospective, open-label, randomized trial was conducted to compare the efficacies of the vasoactive drugs, terlipressin, somatostatin, and octreotide, in patients with cirrhosis and acute variceal bleeding when they were combined with endoscopic therapy. Because we hypothesized that these drugs have the same treatment efficacies, the present study was designed as a noninferiority test. The 5-day treatment success rate was assessed, as determined by the control of bleeding free of rescue treatment, rebleeding, and mortality.

\section{Patients and Methods}

Patients. Eleven medical centers distributed throughout Korea participated in this study. Patients with liver cirrhosis presenting with either hematemesis or melena between October 1, 2006 and May 31, 2010 were assessed for inclusion in this trial after obtaining informed written consent from all potential patients or from their next of kin when patients were unable to give this consent. Inclusion criteria were as follows: (1) liver cirrhosis diagnosed by previous liver biopsy or by compatible clinical, laboratory, and radiologic findings; (2) age between 16 and 75 years; (3) arrival at the hospital within 24 hours after the occurrence of hematemesis and/or melena; (4) clinically significant bleeding, defined as systolic blood pressure (BP) $<100 \mathrm{mmHg}$, postural change in $\mathrm{BP}>20$ $\mathrm{mmHg}$, or pulse rate $>100$ beats $/ \mathrm{min}^{22}$; and (5) patients agreeing to participate in this trial with informed consent. Patients were excluded if they presented any of the following: (1) noncirrhotic PH; (2) a history of endoscopic variceal therapy within 2 weeks before the episode; (3) enrollment during the 6-week period before the index bleed; (4) a history of severe cardiovascular disease, including acute myocardial infarction, atrioventricular block, congestive heart failure, ischemic heart disease, or severe hypertension (systolic BP $>170 \mathrm{mmHg}$ and/or diastolic BP $>100$ $\mathrm{mmHg}$ ); (5) chronic renal failure; (6) advanced hepatocellular carcinoma (HCC) invading portal vein; (7) other malignancy; (8) pregnancy; (9) positive result in a human immunodeficiency virus test; (10) known hypersensitivity to any of the study drugs; or (11) refusal to participate in the study.

This study conformed to the ethical guidelines of the 1975 Declaration of Helsinki and was approved by the ethical committees for human investigations at all

Address reprint requests to: Soon Ho Um, M.D., Ph.D., Division of Gastroenterology and Hepatology, Department of Internal Medicine, Korea University College of Medicine, 126-1, 5-Ga, Anam-Dong, Seongbuk-Gu, Seoul 136-705, Korea. E-mail: umsh@korea.ac.kr; fax: 82-2-953-1943.

Copyright $(2014$ by the American Association for the Study of Liver Diseases.

View this article online at wileyonlinelibrary.com.

DOI 10.1002/hep.27006

Potential conflict of interest: Nothing to report. 
of the enrolled hospitals. The study protocol was registered at www.clinicaltrials.gov (ClinicalTrials.gov identifier: NCT00966355).

Definitions. Time zero (T0) was defined as the time of admission to the first hospital that the patient was taken to. ${ }^{23}$ In patients who presented a clinical sign of bleeding (e.g., hematemesis or melena) during hospitalization for other reasons, the time when the patient noticed the sign was considered as T0. Index bleeding was defined as controlled when there was no hematemesis, hemoglobin $(\mathrm{Hb})$ level was stable without requiring blood transfusions, and vital signs were stable (systolic BP $>100 \mathrm{mmHg}$ and pulse rate $<100$ beats/min) for 24 hours. ${ }^{1}$ Bleeding control time was defined as the beginning time of this 24-hour interval. ${ }^{1}$ Rebleeding was defined as any occurrence of hematemesis or an $\mathrm{Hb}$ decrease with fresh melena after the successful control of initial bleeding. ${ }^{1}$ Mortality was defined as death from any cause within 5 days from T0.

Study Aims. The primary aim was to characterize the three treatment groups of patients regarding the combined endpoint of control of bleeding, avoidance of rescue treatment, prevention of rebleeding, and survival during 5 days of treatment; these parameters were also used in a previous study. ${ }^{9,10}$ Treatment was considered successful when the initial bleeding was controlled without rescue treatment and the patient remained alive without early recurrence of bleeding at 120 hours after T0. The secondary aims were to determine the incidence of active bleeding at the time of the initial endoscopic procedure, the occurrence of related events of bleeding control without rescue treatment, prevention of rebleeding, survival, and the safety profile.

Clinical Evaluation. All patients were managed in the hospital during the 5-day treatment with regular monitoring of the $\mathrm{BP}$ and pulse rate: every 1 hour during the first 12 hours, every 2 hours during 12-24 hours, every 4 hours during 24-48 hours, every 6 hours during 48-120 hours, and every 12 hours after 120 hours. Hb and hematocrit (Hct) levels were measured every 3 hours during the first 12 hours, every 6 hours during 12-48 hours, and then every 24 hours. In addition, routine laboratory tests, such as hematologic parameters, blood chemistry, and urinalysis, were performed regularly. Presence of infectious diseases was investigated with appropriate diagnostic methods. Patients with a fever $>38^{\circ} \mathrm{C}$ that lasted more than 24 hours and leukocytosis with a shift to the left, but without any other evidence of infection, were considered as having possible infections. ${ }^{24}$ Hepatic dysfunc- tion was evaluated according to the Child-Pugh classification $^{25}$ and the Model for End-stage Liver Disease (MELD) score employed by the United Network of Organ Sharing (http://www.unos.org). Stage of HCC was classified according to the modified Union for International Cancer Control classification. ${ }^{26}$

Definitions for the classifications according to type and source of bleeding are described in the Supporting Methods.

Pharmacologic Treatment. Once enrolled, patients were randomly allocated to the following three groups using sealed opaque envelopes numbered according to a table of random numbers: terlipressin; somatostatin; and octreotide groups. Randomization was performed using computer-generated random numbers to allocate patients to the terlipressin, somatostatin, and octreotide groups in a 1:1:1 ratio. Study drugs were administered immediately after randomization as follows: terlipressin (2 mg) by intravenous (IV) bolus followed by $1 \mathrm{mg}$ IV every 6 hours for 5 days, somatostatin $(250 \mu \mathrm{g})$ by IV bolus followed by $250 \mu \mathrm{g} /$ hour continuous infusion for 5 days, and octreotide $(50 \mu \mathrm{g})$ by IV bolus followed by $25 \mu \mathrm{g} /$ hour continuous infusion for 5 days. The vasoactive drug was stopped after endoscopy in cases where bleeding was determined to be unrelated to gastroesophageal varices (GOVs).

Endoscopic Treatments. After initiation of pharmacologic treatment, endoscopic examination was performed as soon as patients had been resuscitated. Endoscopic treatment was applied immediately in patients diagnosed with bleeding from GOVs on endoscopy. EVL and endoscopic variceal obturation (EVO) with cyanoacrylate were considered as the primary therapies for bleeding from esophageal or gastric cardial varices and for bleeding from gastric fundal varices, respectively. ${ }^{14}$ If the primary therapy was not technically feasible, EIS and EVL/EIS were performed for esophageal/cardial varices and for fundal varices, respectively.

In the present study, bleeding from EVs was treated with EVL in $92.0 \%$ of patients, except for minor cases managed by EIS (0.9\%); bleeding from GOV1 were also mainly treated with EVL (71.0\%) followed by EVO (22.6\%), bleeding from GOV2 was principally treated with EVO (72.3\%) followed by EVL (20.0\%), and bleeding from isolated gastric varices type 1 (IGV1) was principally treated with EVO $(79.3 \%)$ followed by EVL (10.3\%).

Detailed information about endoscopic treatments and other treatments are described in the Supporting Methods.

Statistical Analysis. Statistical analyses were performed with the Statistical Package for Social Sciences 
(version 13.0; SPSS, Inc., Chicago, IL). Statistical significance was established at $P<0.05$. All $P$ values were two-tailed. Methods for the sample-size calculation are described in the Supporting Methods. For noninferiority tests of comparing treatment success proportions among terlipressin, somatostatin, and octreotide, $98.3 \%[(1-0.05 / 3) \times 100]$ one-sided confidence intervals (CIs) of proportion difference between pair-wise groups were calculated with Bonferroni's type adjustment for multiple comparisons. Terlipressin was considered as an active control when we compared somatostatin and octreotide with terlipressin. When we compared somatostatin with octreotide, octreotide was considered as an active control for the noninferiority test. If the absolute value of the lower bound of the interval was lower than the noninferiority margin of 0.1 , we concluded that the treatment was noninferior to the control. Data are presented as mean \pm standard deviation or number of patients and percentage values. Categorical and continuous variables were compared with the chi-square test and Student $t$ test, respectively. Multivariate logistic regression analyses were performed to detect independent predictive factors for 5-day treatment failure. To find a compact and predictive model, we first included all the variables significant from univariate logistic regression and selected final predictors using a backward step-wise selection procedure. Patients with an undefined source for bleeding were not included in the multivariate analyses because information on endoscopic findings, such as the source or type of bleeding, was not available in these patients. For the significant variables, adjusted odds ratios (ORs) with two-sided 95\% CIs are presented.

\section{Results}

Patients' Characteristics. In total, 1,160 patients with cirrhosis presenting with hematemesis and/or melena were screened during the study period, of which 1,034 fulfilled the inclusion criteria and were randomly assigned to receive one of the three vasoactive drugs: terlipressin $(\mathrm{n}=345)$, somatostatin $(n=345)$, or octreotide ( $n=344$; Fig. 1$)$. At the initial endoscopic procedure, 209 of the 1,034 enrolled patients were found to have bleeding from lesions other than GOVs, so infusion of the vasoactive drug was discontinued (Fig. 1). Another 33 patients subsequently dropped out of the study because of newly diagnosed advanced HCC, and 12 were lost to followup. Therefore, 780 patients were included in the final analysis: 261 in the terlipressin group, 259 in the somatostatin group, and 260 in the octreotide group (Fig. 1).

Baseline characteristics of the 780 included patients are presented in Table 1 . The study population was male dominant $(85.4 \%)$ and $53 \pm 10$ years of age. Alcoholic liver disease (56.0\%) was the most common cause of liver cirrhosis, followed by chronic hepatitis B $(31.7 \%)$. A previous history of variceal bleeding was found in 340 (43.6\%) patients, of which 150 (41.4\%) had received prophylactic management for rebleeding. HCCs were combined in 82 patients (10.5\%). Infection was noted in 59 patients $(7.6 \%)$ at enrollment, including pneumonia (1.8\%), spontaneous bacterial peritonitis $(1.7 \%)$, sepsis (1.2\%), bronchitis (0.5\%), urinary tract infection $(0.1 \%)$, and possible infection $(2.3 \%)$. Most of the patients belonged to Child-Pugh grades B (42.7\%) and C (32.8\%). Source of bleeding was revealed by endoscopy in 740 of the 780 patients (94.9\%): EVs in 553 (70.9\%), GOV1 in 93 (11.9\%), GOV2 in $65(8.3 \%)$, IGV1 in $29(3.7 \%)$, and undefined in $40(5.1 \%)$ patients in whom endoscopy was unavailable because of hemodynamic instability $(n=28)$ or impaired mental status $(n=12)$. Among 40 patients with undefined source of bleeding, 6 died before rescue therapy and bleeding was controlled with vasoactive drugs alone in 10 . Twenty-four patients were treated with balloon tamponade and 20 of these died within 5 days.

Baseline characteristics, including age, hemodynamic and hematologic parameters, biochemical data, source of bleeding, and other clinical features, did not differ significantly between the three treatment groups (Table 1). Times from onset of hemorrhage to T0, from T0 to commencement of a vasoactive drug, and from $\mathrm{T} 0$ to initial endoscopy were did not differ between the three treatment groups (Table 1).

Outcome of Bleeding. At the time of initial endoscopy, active bleeding was noted in 342 of 740 patients (46.2\%), and its incidence did not differ significantly between the terlipressin, somatostatin, and octreotide groups $(46.0 \%, 46.2 \%$, and $46.5 \%$, respectively; $P=0.748$; Table 1$)$.

Index bleeding was controlled without rescue treatments in 690 of the 780 patients $(88.5 \%)$ at $9.8 \pm 10.3$ hours after T0. Rate of bleeding control without rescue treatment did not differ between the terlipressin, somatostatin, and octreotide groups (89.7\%, 87.6\%, and $88.1 \%$, respectively; $P=0.752$ ). In addition, neither the time from T0 to bleeding control nor the time from the start of the vasoactive drug to bleeding control differed significantly between the treatment groups (Table 2). Among 90 patients in 


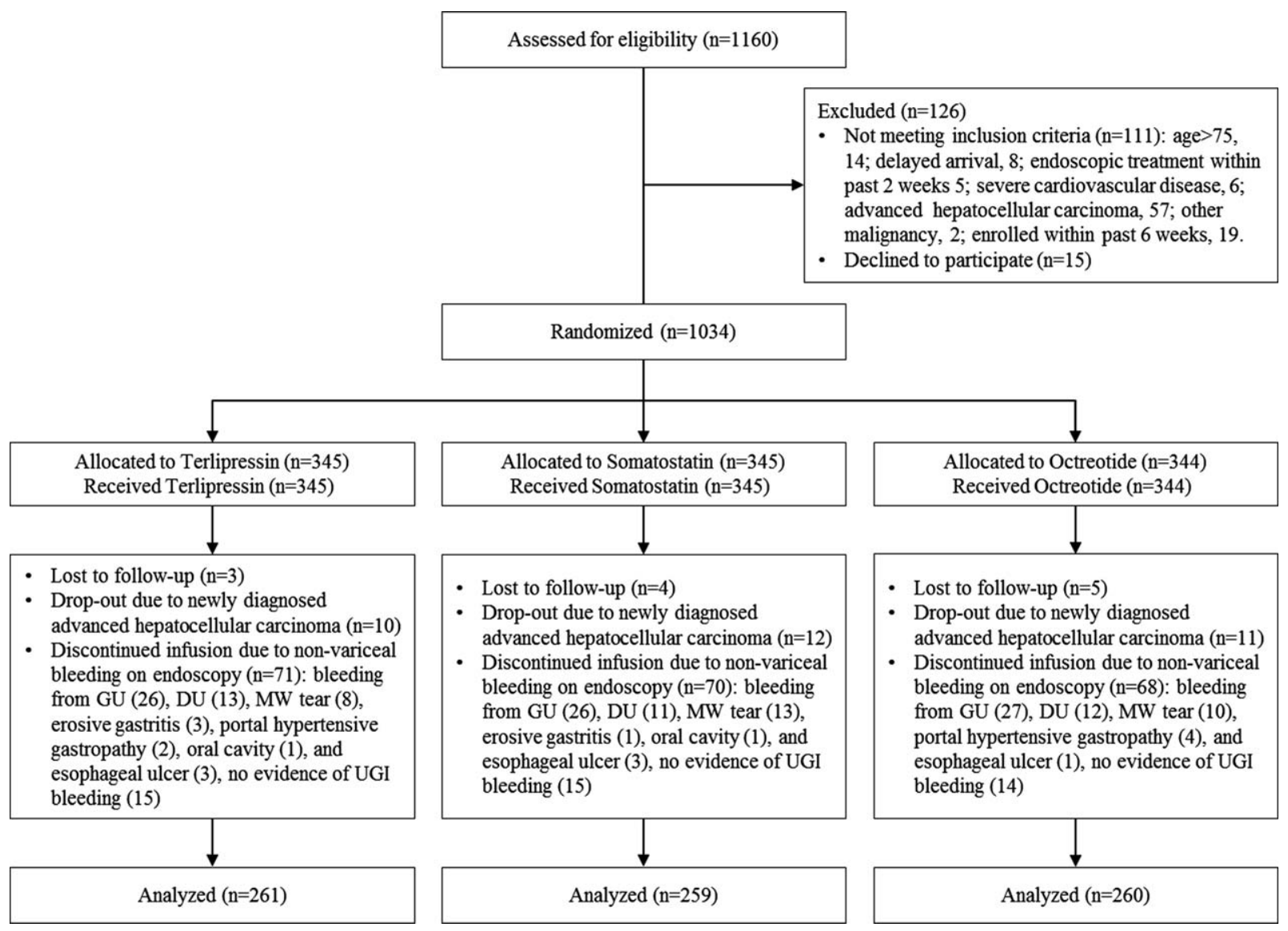

Fig. 1. Flowchart of 1,034 enrolled patients with liver cirrhosis and clinically significant bleeding. GU, gastric ulcer; DU, duodenal ulcer; MW tear, Mallory-Weiss tear; UGI, upper gastrointestinal.

whom bleeding was not controlled by initial therapy, $59(65.6 \%), 3(3.3 \%)$, and $3(3.3 \%)$ were treated with balloon tamponade, transjugular intrahepatic portosystemic shunt (TIPS), and balloon-occluded retrograde transvenous obliteration, respectively, as a rescue therapy. The remaining 25 patients were treated with pharmacological therapy alone because they died before intervention $(\mathrm{n}=9 ; 10.0 \%)$ or refused further treatment $(\mathrm{n}=16 ; 17.8 \%)$.

Of the 690 patients in whom index bleeding was successfully controlled without rescue therapy, 29 (4.2\%) rebled from varices during the 5-day treatment period. However, the rate of recurrent bleeding did not differ significantly between the terlipressin, somatostatin, and octreotide groups $(3.4 \%, 4.8 \%$, and $4.4 \%$, respectively; $P=0.739$ ). Time from control of index bleeding to rebleeding also did not differ significantly between the treatment groups (Table 2 ).

A total of 67 of the 780 patients $(8.6 \%)$ died at $37.0 \pm 29.8$ hours after T0 during the 5-day treatment period. Causes of death were uncontrolled index bleed in 60 patients $(7.7 \%)$, uncontrolled rebleeding in 2 $(0.3 \%)$, liver failure in $3(0.4 \%)$, and sepsis in 2
$(0.3 \%)$. The mortality rate did not differ between the terlipressin, somatostatin, and octreotide groups $(8.0 \%$, $8.9 \%$, and $8.8 \%$, respectively; $P=0.929$; Table 2 ).

Ultimately, 5-day treatment success (i.e., control of bleeding without rescue treatment, rebleeding, or mortality, which was the primary aim) was achieved in 659 of the 780 patients $(84.5 \%)$. The rate of 5-day treatment success did not differ between the terlipressin, somatostatin, and octreotide groups (86.2\%, $83.4 \%$, and $83.8 \%$, respectively; $P=0.636$ ). The absolute value of the lower bound of one-sided $98.3 \%$ CIs of differences in proportion of treatment success between the treatment groups all fulfilled our a priori limit of a noninferiority $(\delta)$ of 0.1 : terlipressin versus somatostatin, 0.095; terlipressin versus octreotide, 0.090; and octreotide versus somatostatin, 0.065. Subgroup analysis was performed according to Child-Pugh grade and cause of bleeding. Treatment outcome between the three groups also did not differ in these analyses (Supporting Tables 1 and 2).

The requirement for a blood transfusion to maintain $\mathrm{Hb}$ levels at $8 \mathrm{~g} / \mathrm{dL}$ or higher did not differ between the terlipressin, somatostatin, and octreotide 
Table 1. Baseline characteristics and the Source and Type of Bleeding in Patients With Variceal Bleeding According to the Type of Vasoactive Drug

\begin{tabular}{|c|c|c|c|c|c|}
\hline Characteristics & All Patients $(n=780)$ & Terlipressin Group $(n=261)$ & Somatostatin Group $(n=259)$ & Octreotide Group ( $n=260$ ) & $P$ Value \\
\hline Age, years & $53.3 \pm 9.6$ & $52.9 \pm 9.2$ & $53.1 \pm 9.7$ & $53.8 \pm 10.0$ & 0.539 \\
\hline Male, n (\%) & $666(85.4)$ & $223(85.4)$ & $216(83.4)$ & 227 (87.3) & 0.539 \\
\hline Cause of liver disease, $\mathrm{n}(\%)$ & & & & & 0.279 \\
\hline Alcohol & $437(56.0)$ & $149(57.1)$ & $157(60.6)$ & $131(50.4)$ & \\
\hline Chronic HBV infection & $247(31.7)$ & $78(29.9)$ & $73(28.2)$ & $96(36.9)$ & \\
\hline Chronic HCV infection & $27(3.5)$ & $9(3.4)$ & $8(3.1)$ & $10(3.8)$ & \\
\hline Alcohol + HBV & $24(3.1)$ & $7(2.7)$ & $11(4.2)$ & $6(2.3)$ & \\
\hline Alcohol + HCV & $12(1.5)$ & $3(1.1)$ & $3(1.2)$ & $6(2.3)$ & \\
\hline $\mathrm{HBV}+\mathrm{HCV}$ & $3(0.4)$ & $0(0)$ & $2(0.8)$ & $1(0.4)$ & \\
\hline Others & $8(1.0)$ & $4(1.5)$ & $1(0.4)$ & $3(1.2)$ & \\
\hline Cryptogenic & $22(2.8)$ & $11(4.2)$ & $4(1.5)$ & $7(2.7)$ & \\
\hline Previous bleeding, n (\%) & $362(46.4)$ & $134(51.3)$ & $111(42.9)$ & $117(45.0)$ & 0.130 \\
\hline Previous variceal bleeding, $\mathrm{n}(\%)$ & $340(43.6)$ & $124(47.5)$ & $105(40.5)$ & $111(42.7)$ & 0.260 \\
\hline HCC, n (\%) & $82(10.5)$ & $27(10.3)$ & $25(9.7)$ & $30(11.5)$ & 0.778 \\
\hline Stage I & $12(1.5)$ & $3(1.1)$ & $6(2.3)$ & $3(1.2)$ & \\
\hline Stage II & $39(5.0)$ & $14(5.4)$ & $11(4.2)$ & $14(5.4)$ & \\
\hline Stage III & $31(4.0)$ & $10(3.8)$ & $8(3.1)$ & $13(5.0)$ & \\
\hline Diabetes, n (\%) & $221(28.3)$ & $67(25.7)$ & $71(27.4)$ & $83(31.9)$ & 0.263 \\
\hline Systolic BP, mmHg & $101.0 \pm 23.1$ & $99.1 \pm 23.7$ & $100.8 \pm 23.2$ & $103.2 \pm 22.3$ & 0.100 \\
\hline Diastolic BP, mmHg & $61.5 \pm 14.4$ & $60.2 \pm 14.8$ & $61.6 \pm 14.7$ & $62.6 \pm 13.6$ & 0.160 \\
\hline Heart rate, beats/min & $104.4 \pm 21.7$ & $105.7 \pm 21.5$ & $102.7 \pm 21.6$ & $104.7 \pm 22.0$ & 0.265 \\
\hline $\mathrm{Hb}, \mathrm{g} / \mathrm{dL}$ & $8.7 \pm 2.2$ & $8.5 \pm 2.2$ & $8.8 \pm 2.2$ & $8.8 \pm 2.2$ & 0.410 \\
\hline Hct, \% & $25.8 \pm 6.2$ & $25.2 \pm 6.2$ & $25.8 \pm 6.0$ & $25.9 \pm 6.4$ & 0.406 \\
\hline Platelet count, $\times 10^{3} / \mathrm{mm}^{3}$ & $105.3 \pm 56.3$ & $100.8 \pm 48.8$ & $109.7 \pm 63.3$ & $105.5 \pm 55.7$ & 0.167 \\
\hline INR & $1.6 \pm 0.5$ & $1.6 \pm 0.6$ & $1.5 \pm 0.4$ & $1.6 \pm 0.5$ & 0.325 \\
\hline BUN, mg/dL & $27.5 \pm 16.7$ & $27.8 \pm 16.9$ & $28.1 \pm 18.3$ & $26.5 \pm 14.6$ & 0.510 \\
\hline Creatinine, mg/dL & $1.0 \pm 0.5$ & $1.0 \pm 0.5$ & $1.0 \pm 0.5$ & $1.1 \pm 0.5$ & 0.869 \\
\hline AST, IU/L & $121.8 \pm 252.3$ & $119.4 \pm 245.7$ & $121.6 \pm 290.5$ & $124.3 \pm 216.3$ & 0.974 \\
\hline ALT, IU/L & $50.3 \pm 102.1$ & $50.0 \pm 138.3$ & $49.0 \pm 72.3$ & $51.9 \pm 83.3$ & 0.943 \\
\hline Bilirubin, mg/dL & $2.7 \pm 3.0$ & $2.9 \pm 3.7$ & $2.7 \pm 2.9$ & $2.6 \pm 2.3$ & 0.430 \\
\hline Albumin, g/dL & $2.8 \pm 0.6$ & $2.8 \pm 0.6$ & $2.8 \pm 0.6$ & $2.9 \pm 0.6$ & 0.118 \\
\hline Child-Pugh score & $8.5 \pm 2.2$ & $8.6 \pm 2.3$ & $8.5 \pm 2.1$ & $8.4 \pm 2.1$ & 0.383 \\
\hline Child-Pugh grade, n (\%) & & & & & 0.659 \\
\hline$A$ & $152(19.5)$ & $49(18.8)$ & $46(17.8)$ & $57(21.9)$ & \\
\hline B & $372(47.7)$ & $121(46.4)$ & $126(48.6)$ & $125(48.1)$ & \\
\hline C & $256(32.8)$ & $91(34.9)$ & $87(33.6)$ & $78(30.0)$ & \\
\hline MELD score & $15.1 \pm 5.6$ & $15.4 \pm 5.9$ & $15.0 \pm 5.3$ & $15.0 \pm 5.6$ & 0.659 \\
\hline Infection, n (\%) & $59(7.6)$ & $19(7.3)$ & $16(6.2)$ & $24(9.2)$ & 0.412 \\
\hline \multicolumn{6}{|l|}{ Time interval, hour } \\
\hline Start of bleeding to TO & $6.8 \pm 6.4$ & $6.8 \pm 6.3$ & $6.8 \pm 6.7$ & $6.8 \pm 6.2$ & 0.998 \\
\hline TO to vasoactive drug & $1.8 \pm 2.0$ & $1.8 \pm 1.9$ & $1.9 \pm 2.3$ & $1.6 \pm 1.7$ & 0.325 \\
\hline TO to endoscopy & $4.5 \pm 4.4$ & $4.6 \pm 4.5$ & $4.7 \pm 4.6$ & $4.3 \pm 3.9$ & 0.526 \\
\hline Source of bleeding & & & & & 0.099 \\
\hline EVs & $553(70.9)$ & $195(74.7)$ & $180(69.5)$ & $178(68.5)$ & \\
\hline GOVs, type 1 & $93(11.9)$ & $23(8.8)$ & $34(13.1)$ & $36(13.8)$ & \\
\hline GOVs, type 2 & $65(8.3)$ & $16(6.1)$ & $29(11.2)$ & $20(7.7)$ & \\
\hline IGVs, type 1 & $29(3.7)$ & $14(5.4)$ & $6(2.3)$ & $9(3.5)$ & \\
\hline Undefined & $40(5.1)$ & $13(5.0)$ & $10(3.9)$ & $17(6.5)$ & \\
\hline Type of bleeding & & & & & 0.748 \\
\hline Active bleeding & $342(43.8)$ & $114(43.7)$ & $115(44.4)$ & $113(43.5)$ & \\
\hline Stigmata & 330 (42.3) & $110(42.1)$ & $108(41.7)$ & $112(43.1)$ & \\
\hline Nonactive, no stigmata & $68(8.7)$ & $24(9.2)$ & $26(10.0)$ & $18(6.9)$ & \\
\hline Undefined & $40(5.1)$ & $13(5.0)$ & $10(3.9)$ & $17(6.5)$ & \\
\hline
\end{tabular}

Abbreviations: ALT, alanine aminotransferase; BUN, blood urea nitrogen; HBV, hepatitis B virus; HCV, hepatitis C virus.

groups during day $1 \quad(3.3 \pm 3.3$ vs. $3.2 \pm 3.2$ vs. $3.0 \pm 3.2$ units; $P=0.605)$ or during the 5 -day treatment period $(4.6 \pm 4.5$ vs. $4.3 \pm 4.1$ vs. $4.3 \pm 5.1$ units; $P=0.732)$.
The cumulative 42 -day mortality rate was $12.0 \%$ in the whole series. There was no significant difference in the 42-day mortality rate between the three groups: terlipressin group, $13.1 \%$; somatostatin group, $11.3 \%$, 
Table 2. Treatment Responses in All Enrolled Patients With Variceal Bleeding

\begin{tabular}{|c|c|c|c|c|c|}
\hline Treatment Response & $\begin{array}{l}\text { All Patients } \\
(\mathrm{n}=\mathbf{7 8 0})\end{array}$ & $\begin{array}{c}\text { Terlipressin } \\
\text { Group }(n=261)\end{array}$ & $\begin{array}{l}\text { Somatostatin } \\
\text { Group }(n=259)\end{array}$ & $\begin{array}{c}\text { Octreotide } \\
\text { Group (n=2 60) }\end{array}$ & $P$ Value \\
\hline Control of index bleeding without rescue therapy, $n(\%)$ & $690(88.5)$ & $234(89.7)$ & $227(87.6)$ & $229(88.1)$ & 0.752 \\
\hline Time interval from T0 to bleeding control, hours & $9.8 \pm 10.3$ & $9.6 \pm 10.7$ & $10.1 \pm 10.1$ & $9.7 \pm 10.2$ & 0.839 \\
\hline $\begin{array}{l}\text { Time interval from commencement of vasoactive } \\
\text { drug to bleeding control, hours }\end{array}$ & $8.0 \pm 10.3$ & $7.8 \pm 10.6$ & $8.2 \pm 10.1$ & $8.1 \pm 10.3$ & 0.899 \\
\hline Patients with rebleeding, $\mathrm{n}(\%)^{*}$ & $29(4.2)$ & $8(3.4)$ & $11(4.8)$ & $10(4.4)$ & 0.739 \\
\hline Time interval from T0 to rebleeding, hours $^{\dagger}$ & $66.8 \pm 23.6$ & $70.0 \pm 27.5$ & $69.0 \pm 19.6$ & $61.9 \pm 26.0$ & 0.730 \\
\hline Time interval from bleeding control to rebleeding, hours ${ }^{\dagger}$ & $62.1 \pm 24.4$ & $64.7 \pm 26.4$ & $64.2 \pm 22.4$ & $57.9 \pm 26.8$ & 0.801 \\
\hline Mortality, n (\%) & $67(8.6)$ & $21(8.0)$ & $23(8.9)$ & $23(8.8)$ & 0.929 \\
\hline Cause of mortality & & & & & 0.920 \\
\hline Uncontrolled index bleeding, $\mathrm{n}(\%)^{* * *}$ & $60(89.6)$ & $19(90.5)$ & $20(87.0)$ & $21(91.3)$ & \\
\hline Uncontrolled rebleeding, $\mathrm{n}(\%)^{\ddagger}$ & $2(3.0)$ & $1(4.8)$ & $1(4.3)$ & $0(0)$ & \\
\hline Liver failure, $\mathrm{n}(\%)^{\ddagger}$ & $3(4.5)$ & $1(4.8)$ & $1(4.3)$ & $1(4.3)$ & \\
\hline Infection, n (\%) ${ }^{\ddagger}$ & $2(3.0)$ & $0(0)$ & $1(4.3)$ & $1(4.3)$ & \\
\hline 5-day treatment success, n (\%) & $659(84.5)$ & $225(86.2)$ & $216(83.4)$ & $218(83.8)$ & 0.636 \\
\hline
\end{tabular}

*Among patients whose index bleeding was successfully controlled.

${ }^{\dagger}$ Among 29 patients with rebleeding.

${ }^{\ddagger}$ Among 67 cases of mortality.

and octreotide group, 11.6\% $(P=0.891$; Supporting Fig. 1).

Adverse Events. Adverse events (AEs) were noted in 57 patients (7.3\%; Table 3). Hyponatremia (defined as a drop of serum $\mathrm{Na}$ level $\geq 5 \mathrm{mmEq}$ from baseline to $<130 \mathrm{mEq} / \mathrm{L}$ ) developed in the terlipressin group (30 of $261 ; 11.5 \%$ ), compared to the somatostatin ( 4 of $259 ; 1.5 \%$ ) and octreotide (3 of $260 ; 1.2 \%$ ) groups $(P<0.001)$. The frequency of other side effects did not differ significantly between these three groups $(P=0.954)$.

Prognostic Factors. Comparison of the baseline characteristics between patients with 5-day treatment success (650 patients) and those with 5-day treatment failure (90 patients) revealed that the proportion of patients with infection at T0, heart rate, international normalized ratio (INR), serum aspartate aminotransferase (AST) and creatinine levels, Child-Pugh score, the proportion of patients with Child-Pugh grade C, MELD score, the proportion of patients with bleeding from gastric varices $(\mathrm{GVs})$, and the proportion of patients with active bleeding were significantly higher in patients with 5-day treatment failure, whereas the systolic BP, diastolic BP, platelet count, and serum albumin level were significantly lower in those patients (Table 4). A multivariate analysis using those variables that were significant in the univariate analysis was performed to define the independent factors for predicting the 5-day treatment failure. This revealed that the independent predictive factors for 5-day treatment failure were low systolic BP at T0, high serum creatinine level, active bleeding in the emergency endoscopy, bleeding from GVs, and Child-Pugh grade C (Supporting Table 3).

\section{Discussion}

This large-scale, multicenter, randomized trial has provided convincing evidence supporting the therapeutic equivalence of three well-known vasoactive drugs as an adjuvant therapy to standard endoscopic treatments in patients with variceal bleeding. This study is the first to compare three drugs simultaneously in a sufficiently large number of patients with variceal hemorrhage. The obtained results indicate that the hemostatic efficacies of terlipressin, somatostatin, and octreotide are the same in patients with clinically significant variceal bleeding when they were administered before standard endoscopic treatments, as recommended in most of the current practice guidelines. Also, the subgroup analysis according to the source of bleeding, which was a strong determinant of treatment outcome, found that treatment responses did not differ between the three drugs. These results are consistent with those of previous studies that analyzed the hemostatic effects of monotherapy with vasoactive drugs. Our study also confirms the results of a recent randomized, double-blind, placebo-controlled trial, which found that terlipressin and octreotide had the same hemostatic efficacy in the setting of combination therapy with EVL in patients with bleeding from EVs. ${ }^{27}$

Several previous randomized trials and meta-analyses have suggested that terlipressin is the only drug that provides a survival benefit, compared to placebo, in patients with variceal bleeding. ${ }^{5,18,19,28}$ This has prompted some researchers to recommend terlipressin as the first choice and somatostatin or octreotide as the second choice. ${ }^{17,28}$ However, many other studies that have compared the clinical efficacies of different 
Table 3. AEs in All Enrolled Patients With Variceal Bleeding

\begin{tabular}{lcccc}
\hline AEs & $\begin{array}{c}\text { Terlipressin } \\
\text { Group }\end{array}$ & $\begin{array}{c}\text { Somatostatin } \\
\text { Group }\end{array}$ & $\begin{array}{c}\text { Octreotide } \\
\text { Group }\end{array}$ & Total \\
\hline Fever & 0 & 0 & 1 & 1 \\
Diarrhea & 0 & 0 & 2 & 2 \\
Nausea & 0 & 1 & 0 & 1 \\
Vomiting & 1 & 0 & 0 & 1 \\
Abdominal pain & 4 & 3 & 1 & 8 \\
Chest pain & 0 & 0 & 1 & 1 \\
Change in EKG & 1 & 3 & 2 & 6 \\
Hyponatremia & 30 & 4 & 3 & 37 \\
Total & 36 & 11 & 10 & 57 \\
\hline
\end{tabular}

Abbreviation: EKG, electrocardiography.

types of vasoactive drugs as monotherapy found no differences in mortality rates. ${ }^{16}$ Furthermore, the present study, which included a sufficiently large number of patients, convincingly shows that mortality rate does not differ significantly between terlipressin and somatostatin or octreotide also in the setting of combination therapy with endoscopic treatment. Therefore, any of the three splanchnic vasoconstrictors (i.e., terlipressin, somatostatin, and octreotide) could be employed equivalently as an adjuvant therapy to standard endoscopic therapies to control gastroesophageal variceal hemorrhage. Meanwhile, prevalence of hyponatremia was more frequent in the terlipressin group, which was similar with a previous study. ${ }^{29}$

In our study, low systolic BP at T0, high serum creatinine level, active bleeding in the emergency endoscopy, bleeding from GVs, and Child-Pugh grade C were the independent predictive factors for 5-day treatment failure. These results are very similar to previous studies that have suggested a higher Child-Pugh class, ${ }^{1,30,31}$ shock, ${ }^{8}$ active bleeding, ${ }^{8,30}$ and high serum creatinine level $(\geq 1.0 \mathrm{mg} / \mathrm{dL})^{31}$ to be significant factors for a poor prognosis.

It might be argued that the treatment responses were worse in our study than in previous studies. ${ }^{1,27}$ This can be explained by differences in the study designs and patient populations. Comparison of a previous large prospective study ${ }^{1}$ with our study indicated no differences in the 5 -day rebleeding rate $(3.9 \%$ vs. $4.2 \%)$, 5-day mortality (9.2\% vs. $8.6 \%)$, and overall 5 -day failure rate $(14.8 \%$ vs. $15.5 \%)$, but the hemostasis failure rate was higher in our study $(1.5 \%$ vs. $11.5 \%)$. This might be, at least partly, attributable to stricter criteria being applied in the present study for successful hemostasis, which did not include cases with bleeding controlled by rescue therapies. This discrepancy might also be explained by the proportion of patients with EV bleeding differing significantly between the present study $(74.7 \%)$ and the previous
Table 4. Comparison of Baseline Characteristics and Endoscopic Findings Between Patients With 5-Day Treatment Success and Those With 5-Day Treatment Failure Among Patients With a Defined Source of Bleeding

\begin{tabular}{|c|c|c|c|}
\hline Characteristics & $\begin{array}{c}\text { Patients With } \\
\text { 5-day Treatment } \\
\text { Success }(n=650)\end{array}$ & $\begin{array}{c}\text { Patients With } \\
\text { 5-day Treatment } \\
\text { Failure }(n=90)\end{array}$ & $P$ Value \\
\hline Age, years & $53.1 \pm 9.8$ & $54.0 \pm 8.6$ & 0.427 \\
\hline Sex & & & 0.054 \\
\hline Male, n (\%) & $550(84.6)$ & $83(92.2)$ & \\
\hline Female, $n(\%)$ & $100(15.4)$ & $7(7.8)$ & \\
\hline Cause of liver disease, $n(\%)$ & & & 0.515 \\
\hline Alcohol & $364(56.0)$ & $49(54.4)$ & \\
\hline Chronic HBV infection & $201(30.9)$ & $34(37.8)$ & \\
\hline Chronic HCV infection & $24(3.7)$ & $2(2.2)$ & \\
\hline Alcohol + HBV & $21(3.2)$ & $2(2.2)$ & \\
\hline Alcohol + HCV & $10(1.5)$ & $1(1.1)$ & \\
\hline $\mathrm{HBV}+\mathrm{HCV}$ & $2(0.3)$ & $1(1.1)$ & \\
\hline Others & $7(1.1)$ & $1(1.1)$ & \\
\hline Cryptogenic & $21(3.2)$ & $0(0.0)$ & \\
\hline Alcohol drinking & & & 0.317 \\
\hline No, n (\%) & $232(35.7)$ & 37 (41.1) & \\
\hline Yes, n (\%) & $418(64.3)$ & $53(58.9)$ & \\
\hline Previous variceal bleeding & & & 0.055 \\
\hline No, n (\%) & $373(57.4)$ & $42(46.7)$ & \\
\hline Yes, n (\%) & $277(46.7)$ & $48(53.3)$ & \\
\hline $\mathrm{HCC}$ & & & 0.515 \\
\hline No, n (\%) & $585(90.0)$ & $79(87.8)$ & \\
\hline Yes, n (\%) & $65(10.0)$ & $11(12.2)$ & \\
\hline Diabetes & & & 0.657 \\
\hline No, n (\%) & $462(71.1)$ & 66 (73.3) & \\
\hline Yes, n (\%) & $188(28.9)$ & $24(26.7)$ & \\
\hline Infection at T0 & & & 0.028 \\
\hline No, $n(\%)$ & $611(94.0)$ & $79(87.8)$ & \\
\hline Yes, n (\%) & $39(6.0)$ & $11(12.2)$ & \\
\hline Systolic BP, mmHg & $103.1 \pm 22.8$ & $90.7 \pm 21.0$ & $<0.001$ \\
\hline Diastolic BP, mm Hg & $62.6 \pm 13.9$ & $55.7 \pm 14.5$ & $<0.001$ \\
\hline Heart rate, beats/min & $103.8 \pm 20.4$ & $111.0 \pm 28.2$ & 0.021 \\
\hline $\mathrm{Hb}, \mathrm{g} / \mathrm{dL}$ & $8.7 \pm 2.2$ & $8.4 \pm 1.9$ & 0.183 \\
\hline Platelet count, $\times 10^{3} / \mathrm{mm}^{3}$ & $105.7 \pm 51.7$ & $92.5 \pm 57.3$ & 0.025 \\
\hline INR & $1.5 \pm 0.4$ & $1.7 \pm 0.5$ & 0.007 \\
\hline AST, IU/L & $110.3 \pm 246.8$ & $163.5 \pm 234.8$ & 0.049 \\
\hline ALT, IU/L & $47.2 \pm 104.0$ & $70.2 \pm 103.1$ & 0.051 \\
\hline Bilirubin, mg/dL & $2.6 \pm 2.8$ & $3.2 \pm 4.0$ & 0.068 \\
\hline Albumin, g/dL & $2.9 \pm 0.6$ & $2.7 \pm 0.6$ & 0.019 \\
\hline Glucose, mg/dL & $165.9 \pm 84.1$ & $148.0 \pm 87.6$ & 0.066 \\
\hline Cholesterol, mg/dL & $109.8 \pm 35.6$ & $107.9 \pm 39.0$ & 0.639 \\
\hline BUN, mg/dL & $26.9 \pm 15.4$ & $28.0 \pm 17.9$ & 0.578 \\
\hline Creatinine, $\mathrm{mg} / \mathrm{dL}$ & $1.0 \pm 0.4$ & $1.3 \pm 0.6$ & $<0.001$ \\
\hline Child-Pugh score & $8.3 \pm 2.1$ & $9.4 \pm 2.4$ & $<0.001$ \\
\hline Child-Pugh grade, n (\%) & & & $<0.001$ \\
\hline Grade A or B & $471(72.5)$ & $41(45.6)$ & \\
\hline Grade C & $179(27.5)$ & $49(54.4)$ & \\
\hline MELD score & $14.4 \pm 5.0$ & $17.7 \pm 6.6$ & $<0.001$ \\
\hline Source of bleeding, n (\%) & & & $<0.001$ \\
\hline EVs or GOV1 & $582(89.5)$ & $64(71.1)$ & \\
\hline GOV2 or IGV1 & $68(10.5)$ & $26(28.9)$ & \\
\hline Type of bleeding, n (\%) & & & $<0.001$ \\
\hline Nonactive & $371(57.1)$ & $27(30.0)$ & \\
\hline Active & $279(42.9)$ & $63(70.0)$ & \\
\hline Type of vasoactive drug & & & 0.472 \\
\hline Terlipressin & $222(34.2)$ & $26(28.9)$ & \\
\hline Somatostatin & $214(32.9)$ & $35(38.9)$ & \\
\hline Octreotide & $214(32.9)$ & $29(32.2)$ & \\
\hline
\end{tabular}

Abbreviations: ALT, alanine aminotransferase; BUN, blood urea nitrogen; HBV, hepatitis $B$ virus; $H C V$, hepatitis $C$ virus. 
study $(88.4 \%){ }^{1}$ This explanation is supported by the present finding of the bleeding control rate in patients being significantly lower for bleeding from GOV2 or IGV1 than for bleeding from EV or GOV1. Similarly, in a recent randomized trial that compared terlipressin and octreotide in patients with bleeding from EV as an adjuvant therapy with EVL, bleeding from EV was controlled in 318 of 324 patients $(98.1 \%)$ and only $17(5.2 \%)$ died. ${ }^{27}$ Those rates for bleeding control and survival rates were somewhat higher than our results. However, the included populations differed significantly between the two studies: (1) The previous study enrolled patients with bleeding from EV, and excluded those with bleeding from GVs, ${ }^{27}$ and (2) the proportions of patients with active bleeding (67 of 324) and low systolic BP $(112.7 \pm 20.0$ and $115.0 \pm 19.5$ $\mathrm{mmHg}$ in the terlipressin and octreotide groups, respectively) ${ }^{27}$ were lower than in the present study.

Current practice guidelines recommend the use of vasoactive drugs in patients with potential variceal bleeding, even preceding endoscopic confirmation. ${ }^{12-14}$ Therefore, we enrolled all patients with potential variceal bleeding, started the study drug according to the randomization, and subsequently performed endoscopy. Therefore, many (approximately 20\%) patients with bleeding from other sources of bleeding were excluded after endoscopy. Furthermore, the source of bleeding could not be defined in some patients because of hemodynamic instability or a lack of cooperation associated with altered mentality preventing endoscopy being performed. Most previous studies included patients with variceal bleeding only when this was confirmed by endoscopy. However, because it is often difficult to perform endoscopy in critically ill patients with massive variceal bleeding, excluding these patients could represent a selection bias and result in overestimation of treatment response. We avoided this bias by enrolling these patients and herein present the results in all enrolled patients as well as those with a defined source of bleeding.

There are some limitations in our study. First, our study was not double blinded, and if it had been double blinded, our results may have more strength. Second, randomization was not stratified according to the source of bleeding in this study, although it is already well known that the prognosis differs significantly between patients with esophageal variceal bleeding and those with GVs. Stratification was not possible in this study because patients were randomized before diagnostic endoscopy depending on the protocol. However, the proportion of patients finally did not differ according to the source of bleeding, and treatment outcomes were comparable between the three treatment groups even in the subgroup analysis according to the source of bleeding (Supporting Table 1). Last, in this study, somatostatin was infused at a dose of $250 \mu \mathrm{g} /$ hour. A previous study suggested that higher doses of somatostatin infusion $(500 \mu \mathrm{g} /$ hour $)$ achieved a higher rate of hemostasis and a lower rate of mortality in patients with cirrhosis with active variceal bleeding at emergency endoscopy. ${ }^{32}$ Therefore, further studies are needed to confirm the optimal dose of somatostatin in patients with acute variceal bleeding, although we obtained similar outcomes with a lower dose of somatostatin, compared to other vasoactive agents, in the present study.

In conclusion, treatment response in patients with gastroesophageal variceal hemorrhage does not differ significantly between terlipressin, somatostatin, and octreotide when they are administered in combination with endoscopic therapy. Therefore, any of these drugs could be used equivalently as an adjuvant therapy to standard endoscopic treatments to control gastroesophageal variceal hemorrhage. A low systolic BP at T0, high serum creatinine level, active bleeding in the emergency endoscopy, bleeding from GVs, and Child-Pugh grade $\mathrm{C}$ are the independent predictive factors for 5-day treatment failure. Other treatment options, such as TIPS, could be considered in patients with a high risk of treatment failure.

Acknowledgment: The authors thank Professor Vijay Shah for helpful discussions on the results.

\section{References}

1. D’Amico G, De Franchis R. Upper digestive bleeding in cirrhosis. Post-therapeutic outcome and prognostic indicators. HePatology 2003; 38:599-612.

2. McCormick PA, O’Keefe C. Improving prognosis following a first variceal haemorrhage over four decades. Gut 2001;49:682-685.

3. Baik SK, Jeong PH, Ji SW, Yoo BS, Kim HS, Lee DK, et al. Acute hemodynamic effects of octreotide and terlipressin in patients with cirrhosis: a randomized comparison. Am J Gastroenterol 2005;100:631-635.

4. Bosch J, Kravetz D, Rodes J. Effects of somatostatin on hepatic and systemic hemodynamics in patients with cirrhosis of the liver: comparison with vasopressin. Gastroenterology 1981;80:518-525.

5. D'Amico G, Pagliaro L, Bosch J. The treatment of portal hypertension: a meta-analytic review. Hepatology 1995;22:332-354.

6. Wells M, Chande N, Adams P, Beaton M, Levstik M, Boyce E, Mrkobrada M. Meta-analysis: vasoactive medications for the management of acute variceal bleeds. Aliment Pharmacol Ther 2012;35:1267-1278.

7. Banares R, Albillos A, Rincon D, Alonso S, Gonzalez M, Ruiz-delArbol L, et al. Endoscopic treatment versus endoscopic plus pharmacologic treatment for acute variceal bleeding: a meta-analysis. HePatology 2002;35:609-615.

8. Villanueva C, Ortiz J, Sabat M, Gallego A, Torras X, Soriano G, et al. Somatostatin alone or combined with emergency sclerotherapy in the treatment of acute esophageal variceal bleeding: a prospective randomized trial. Hepatology 1999;30:384-389.

9. Sung JJ, Chung SC, Yung MY, Lai CW, Lau JY, Lee YT, et al. Prospective randomised study of effect of octreotide on rebleeding from oesophageal varices after endoscopic ligation. Lancet 1995;346:1666-1669. 
10. Besson I, Ingrand P, Person B, Boutroux D, Heresbach D, Bernard P, et al. Sclerotherapy with or without octreotide for acute variceal bleeding. N Engl J Med 1995;333:555-560.

11. Avgerinos A, Nevens F, Raptis S, Fevery J. Early administration of somatostatin and efficacy of sclerotherapy in acute oesophageal variceal bleeds: the European Acute Bleeding Oesophageal Variceal Episodes (ABOVE) randomised trial. Lancet 1997;350:1495-1499.

12. Garcia-Tsao G, Bosch J. Management of varices and variceal hemorrhage in cirrhosis. N Engl J Med 2010;362:823-832.

13. Garcia-Tsao G, Sanyal AJ, Grace ND, Carey W. Prevention and management of gastroesophageal varices and variceal hemorrhage in cirrhosis. Hepatology 2007;46:922-938.

14. de Franchis R. Evolving consensus in portal hypertension. Report of the Baveno IV consensus workshop on methodology of diagnosis and therapy in portal hypertension. J Hepatol 2005;43:167-176.

15. Lo GH, Lai KH, Cheng JS, Lin CK, Huang JS, Hsu PI, Chiang HT. Emergency banding ligation versus sclerotherapy for the control of active bleeding from esophageal varices. HePATOLOGY 1997;25:1101-1104.

16. Villanueva C, Piqueras M, Aracil C, Gomez C, Lopez-Balaguer JM, Gonzalez B, et al. A randomized controlled trial comparing ligation and sclerotherapy as emergency endoscopic treatment added to somatostatin in acute variceal bleeding. J Hepatol 2006;45:560-567.

17. Abraldes JG, Bosch J. The treatment of acute variceal bleeding. J Clin Gastroenterol 2007;41(Suppl 3):S312-S317.

18. Soderlund C, Magnusson I, Torngren S, Lundell L. Terlipressin (triglycyl-lysine vasopressin) controls acute bleeding oesophageal varices. A double-blind, randomized, placebo-controlled trial. Scand J Gastroenterol 1990;25:622-630.

19. Levacher S, Letoumelin P, Pateron D, Blaise M, Lapandry C, Pourriat JL. Early administration of terlipressin plus glyceryl trinitrate to control active upper gastrointestinal bleeding in cirrhotic patients. Lancet 1995; 346:865-868.

20. Goulis J, Burroughs AK. Role of vasoactive drugs in the treatment of bleeding oesophageal varices. Digestion 1999;60(Suppl 3):25-34.

21. Escorsell A, Bandi JC, Andreu V, Moitinho E, Garcia-Pagan JC, Bosch J, et al. Desensitization to the effects of intravenous octreotide in cirrhotic patients with portal hypertension. Gastroenterology 2001;120: 161-169.

22. de Franchis R. Developing consensus in portal hypertension. J Hepatol 1996;25:390-394.

23. de Franchis R, Pascal JP, Ancona E, Burroughs AK, Henderson M, Fleig $\mathrm{W}$, et al. Definitions, methodology and therapeutic strategies in portal hypertension. A Consensus Development Workshop, Baveno, Lake Maggiore, Italy, April 5 and 6, 1990. J Hepatol 1992;15:256-261.

24. Pauwels A, Mostefa-Kara N, Debenes B, Degoutte E, Levy VG. Systemic antibiotic prophylaxis after gastrointestinal hemorrhage in cirrhotic patients with a high risk of infection. Hepatology 1996;24:802-806.

25. Pugh RN, Murray-Lyon IM, Dawson JL, Pietroni MC, Williams R. Transection of the oesophagus for bleeding oesophageal varices. $\mathrm{Br}$ J Surg 1973;60:646-649.

26. Ueno S, Tanabe G, Nuruki K, Hamanoue M, Komorizono Y, Oketani M, et al. Prognostic performance of the new classification of primary liver cancer of Japan (4th edition) for patients with hepatocellular carcinoma: a validation analysis. Hepatol Res 2002;24: 395-403.

27. Abid S, Jafri W, Hamid S, Salih M, Azam Z, Mumtaz K, et al. Terlipressin vs. octreotide in bleeding esophageal varices as an adjuvant therapy with endoscopic band ligation: a randomized double-blind placebo-controlled trial. Am J Gastroenterol 2009;104:617-623.

28. Ioannou G, Doust J, Rockey DC. Terlipressin for acute esophageal variceal hemorrhage. Cochrane Database Syst Rev 2003:CD002147.

29. Sola E, Lens S, Guevara M, Martin-Llahi M, Fagundes C, Pereira G, et al. Hyponatremia in patients treated with terlipressin for severe gastrointestinal bleeding due to portal hypertension. Hepatology 2010;52: $1783-1790$

30. Feu F, Ruiz del Arbol L, Banares R, Planas R, Bosch J. Double-blind randomized controlled trial comparing terlipressin and somatostatin for acute variceal hemorrhage. Variceal Bleeding Study Group. Gastroenterology 1996;111:1291-1299.

31. Augustin S, Altamirano J, Gonzalez A, Dot J, Abu-Suboh M, Armengol JR, et al. Effectiveness of combined pharmacologic and ligation therapy in high-risk patients with acute esophageal variceal bleeding. Am J Gastroenterol 2011;106:1787-1795.

32. Moitinho E, Planas R, Banares R, Albillos A, Ruiz-del-Arbol L, Galvez C, et al. Multicenter randomized controlled trial comparing different schedules of somatostatin in the treatment of acute variceal bleeding. J Hepatol 2001;35:712-8.

\section{Supporting Information}

Additional Supporting Information may be found in the online version of this article at the publisher's website. 